\title{
Asymptotics for some nonlinear elliptic systems in Maxwell-Chern-Simons vortex theory
}

\author{
Tonia Ricciardi* \\ Dipartimento di Matematica e Applicazioni \\ Università di Napoli Federico II \\ Via Cintia \\ 80126 Naples, Italy \\ fax: +39081 675665 \\ e-mail: tonia.ricciardi@unina.it
}

\begin{abstract}
We provide a unified proof of the asymptotics of the self-dual MaxwellChern-Simons vortices, as the Maxwell term is neglected, in both the $U(1)$ and $C P(1)$ case. This result is achieved by identifying and analyzing a suitable class of nonlinear elliptic systems with exponential type nonlinearities.
\end{abstract}

KEY WORDS: nonlinear elliptic system, Chern-Simons vortex theory

MCS 2000 SUBJECT CLASSIFICATION: 35J60

\section{Introduction and main result}

The vortex solutions for the $U(1)$ Maxwell-Chern-Simons model introduced in [9], correspond to (distributional) solutions $(\widetilde{u}, v)$ for the system:

$$
\begin{array}{ll}
-\Delta \widetilde{u}=\varepsilon^{-1}\left(v-e^{\widetilde{u}}\right)-4 \pi \sum_{j=1}^{n} \delta_{p_{j}} & \text { on } \Sigma \\
-\Delta v=\varepsilon^{-1}\left\{e^{\widetilde{u}}(1-v)-\varepsilon^{-1}\left(v-e^{\widetilde{u}}\right)\right\} & \text { on } \Sigma,
\end{array}
$$

where $\Sigma$ is a compact Riemannian 2-manifold without boundary, $n \geq 0$ is an integer, $p_{j} \in \Sigma$ for $j=1, \ldots, n, \Delta$ denotes the Laplace-Beltrami operator and $\varepsilon>0$ a constant. We shall be interested in the asymptotic behavior of solutions when $\varepsilon \rightarrow 0$.

Physically, $e^{\widetilde{u}}$ represents a density of particles; it vanishes exactly at the points $p_{j}, j=1, \ldots, n$ (the vortex points). The function $v$ is a neutral scalar field and $\varepsilon>0$ is the coupling constant for the Maxwell term. In particular, letting $\varepsilon \rightarrow 0$ corresponds to dropping the Maxwell term in the Lagrangian.

The limit $\varepsilon \rightarrow 0$ is meaningful in view of the following

\footnotetext{
*Partially supported by PRIN 2000 "Variational Methods and Nonlinear Differential Equations"
} 
Theorem 1.1 ([13]). If $\varepsilon 4 \pi n /|\Sigma|$ is sufficiently small, then there exist at least two solutions for (1) $-(2)$.

The proof of Theorem 1.1 is variational. The two solutions are obtained as a local minimum and a mountain pass for a suitable functional. We refer to [13] for the detailed proof.

By a formal analysis of (1)-(2), we expect that as $\varepsilon \rightarrow 0, e^{u}$ should converge to a solution $u_{\infty}$ for the equation

$$
-\Delta u_{\infty}=e^{u_{\infty}}\left(1-e^{u_{\infty}}\right)-4 \pi \sum_{j=1}^{n} \delta_{p_{j}} \quad \text { on } \Sigma .
$$

We observe that solutions for (3) correspond to vortex solutions for the ChernSimons model introduced in [7] and [6]. In [11] we provided a rigorous proof of this formal argument, in any relevant norm. Namely, we showed

Theorem $1.2([\mathbf{1 1}])$. Suppose $(u, v)$ are (distributional) solutions for (1)-(2) with $\varepsilon \rightarrow 0$. Then there exists a solution $u_{\infty}$ for the equation (3) such that, up to subsequences, $\left(e^{\widetilde{u}}, v\right) \rightarrow\left(e^{u_{\infty}}, e^{u_{\infty}}\right)$ in $C^{h}(\Sigma) \times C^{h}(\Sigma)$, for any $h \geq 0$.

Note that $e^{\widetilde{u}}, e^{u_{\infty}}$ are smooth. Theorem 1.2 completed our previous convergence result obtained with Tarantello [13], where the asymptotics for $v$ was established in the $L^{2}$-sense only. See also Chae and Kim [2].

At this point it is natural to seek a more general class of systems which exhibit an asymptotic behavior as in Theorem 1.2. A further motivation to this question is provided by the analysis of the $C P(1)$ Maxwell-Chern-Simons model in [3]. In [3] the authors analyze an elliptic system, whose solutions correspond to vortex solutions for the self-dual $C P(1)$ Maxwell-Chern-Simons model introduced in [4]. Their system (in a special case) is given by:

$$
\begin{array}{ll}
\Delta U=2 Q\left(-V+S-\frac{1-e^{U}}{1+e^{U}}\right)+4 \pi \sum_{j=1}^{n} \delta_{p_{j}} & \text { on } \Sigma \\
\Delta V=-4 Q^{2}\left(-V+S-\frac{1-e^{U}}{1+e^{U}}\right)+Q \frac{4 e^{U}}{\left(1+e^{U}\right)^{2}} V & \text { on } \Sigma
\end{array}
$$

where $\Sigma$ and $p_{1}, \ldots, p_{n}$ are as in (1)-(2), $U, V$ are the unknown functions and $S \in \mathbb{R}, Q>0$ are given constants. They prove the existence of at least one solution for (4)-(5); furthermore, they derive an asymptotic behavior as $Q \rightarrow$ $+\infty$ analogous to that of system (1)-(2).

With this motivation, we consider (distributional) solutions $(\widetilde{u}, v)$ for the system:

$$
\begin{aligned}
& -\Delta \widetilde{u}=\varepsilon^{-1}\left(v-f\left(e^{\widetilde{u}}\right)\right)-4 \pi \sum_{j=1}^{n} \delta_{p_{j}} \quad \text { on } \Sigma \\
& -\Delta v=\varepsilon^{-1}\left[f^{\prime}\left(e^{\widetilde{u}}\right) e^{\widetilde{u}}(s-v)-\varepsilon^{-1}\left(v-f\left(e^{\widetilde{u}}\right)\right)\right] \quad \text { on } \Sigma .
\end{aligned}
$$

Here $\Sigma$ and $p_{1}, \ldots, p_{n}$ are as in (1)-(2), $f=f(t), t \geq 0$ is smooth and strictly increasing, $s \in \mathbb{R}$ satisfies $f(0)<s<\sup _{t>0} f(t)$. Without loss of generality, we assume $\operatorname{vol} \Sigma=1$. 
Clearly, when $f(t)=t$ and $s=1$, system (6)-(7) reduces to (1)-(2). On the other hand, setting $v:=V-S, s:=-S, \varepsilon^{-1}:=2 Q$, system (6)-(7) reduces to system (4)-(5) with $f$ defined by $f(t)=(t-1) /(t+1)$.

By a formal analysis of (6)-(7) we expect that, up to subsequences, $(\widetilde{u}, v)$ should converge to $\left(\widetilde{u}_{\infty}, f\left(e^{\widetilde{u}_{\infty}}\right)\right)$, where $\widetilde{u}_{\infty}$ is a solutions for the equation for the equation:

$$
-\Delta \widetilde{u}_{\infty}=f^{\prime}\left(e^{\widetilde{u}_{\infty}}\right) e^{\widetilde{u}_{\infty}}\left(s-f\left(e^{\widetilde{u}_{\infty}}\right)\right)-4 \pi \sum_{j=1}^{n} \delta_{p_{j}} \quad \text { on } \Sigma .
$$

Our main result states that this is indeed the case, with respect to any relevant norm:

Theorem $1.3([\mathbf{1 2}])$. Let $(\widetilde{u}, v)$ be (distributional) solutions to (6)-(7), with $\varepsilon \rightarrow 0$. There exists a (distributional) solution $\widetilde{u}_{\infty}$ to (8) such that a subsequence, still denoted $(\widetilde{u}, v)$, satisfies:

$$
\left(e^{\widetilde{u}}, v\right) \rightarrow\left(e^{\widetilde{u}_{\infty}}, f\left(e^{\widetilde{u}_{\infty}}\right)\right) \quad \text { in } C^{h}(\Sigma) \times C^{h}(\Sigma), \quad \forall h \geq 0 .
$$

In the rest of this note we shall outline the proof of Theorem 1.3. The detailed proof is contianed in [12], although some arguments are proved here in a simpler form. Henceforth we denote by $C>0$ a general constant independent

of $\varepsilon$, which may vary from line to line. Unless otherwise specified, all equations are defined on $\Sigma$ and all integrals are taken over $\Sigma$ with respect to the Lebesgue measure.

\section{Proof of Theorem 1.3}

In order to work in suitable Sobolev spaces, it is standard (see [14]) to define a "Green's function" $u_{0}$, solution for the problem

$$
\begin{aligned}
& -\Delta u_{0}=4 \pi\left(n-\sum_{j=1}^{n} \delta_{p_{j}}\right) \quad \text { on } \Sigma \\
& \int_{\Sigma} u_{0}=0
\end{aligned}
$$

(see [1] for the unique existence of $u_{0}$ ). Setting $\widetilde{u}=u_{0}+u$, we obtain the equivalent system for $(u, v) \in H^{1}(\Sigma) \times H^{1}(\Sigma)$ :

$$
\begin{aligned}
& -\Delta u=\varepsilon^{-1}\left(v-f\left(e^{u_{0}+u}\right)\right)-4 \pi n \quad \text { on } \Sigma \\
& -\Delta v=\varepsilon^{-1}\left[f^{\prime}\left(e^{u_{0}+u}\right) e^{u_{0}+u}(s-v)-\varepsilon^{-1}\left(v-f\left(e^{u_{0}+u}\right)\right)\right] \quad \text { on } \Sigma,
\end{aligned}
$$

where $e^{u_{0}}$ is smooth.

The proof is obtained by a priori estimates and an inductive argument. It will be convenient to introduce the spaces $X^{k}:=H^{k} \cap L^{\infty}$. By the following inequality, which follows from the well-known Sobolev-Gagliardo-Nirenberg inequality (see e.g. [10]):

$$
\left\|D^{j} u\right\|_{L^{2 k / j}} \leq C\left\|D^{k} u\right\|_{L^{2}}^{j / k}\|u\|_{L^{\infty}}^{1-j / k} \quad \forall u \in C^{\infty}(\Sigma),
$$


$X^{k}$ is a Banach algebra for every $k \geq 0$, i.e.,

$$
\left\|u_{1} u_{2}\right\|_{X^{k}} \leq C\left\|u_{1}\right\|_{X^{k}}\left\|u_{2}\right\|_{X^{k}} .
$$

It will also be convenient to set

$$
w:=\varepsilon^{-1}\left(v-f\left(e^{u_{0}+u}\right)\right)
$$

and to consider $w$ as a third unknown function. Then the triple $(u, v, w)$ satisfies a system of the following simple form:

$$
\begin{aligned}
& -\Delta u=w-4 \pi n \\
& -\varepsilon^{2} \Delta v+[1+\varepsilon c(x, u)] v=F_{\varepsilon}(x, u) \\
& -\varepsilon^{2} \Delta w+[1+\varepsilon c(x, u)] w=G_{\varepsilon}(x, u, v, \nabla u)
\end{aligned}
$$

where

$$
\begin{aligned}
c(x, u) & =f^{\prime}\left(e^{u_{0}+u}\right) e^{u_{0}+u} \\
F_{\varepsilon}(x, u) & =f\left(e^{u_{0}+u}\right)+s \varepsilon f^{\prime}\left(e^{u_{0}+u}\right) e^{u_{0}+u} \\
G_{\varepsilon}(x, u, v, \nabla u) & =f^{\prime}\left(e^{u_{0}+u}\right) e^{u_{0}+u}(s-v) \\
& +\varepsilon\left(f^{\prime \prime}\left(e^{u_{0}+u}\right) e^{u_{0}+u}+f^{\prime}\left(e^{u_{0}+u}\right)\right) e^{u_{0}+u}\left|\nabla\left(u_{0}+u\right)\right|^{2} .
\end{aligned}
$$

Furthermore, using (11), we obtain:

Lemma 2.1. Let $F \in C^{\infty}(\Sigma \times \mathbb{R}), G \in C^{\infty}\left(\Sigma \times \mathbb{R} \times \mathbb{R} \times \mathbb{R}^{2}\right)$. Then for all $k \geq 0$ there exists constants $C_{k}=C_{k}\left(\|u\|_{L^{\infty}}\right), C_{k}^{\prime}=C_{k}^{\prime}\left(\|u\|_{L^{\infty}},\|v\|_{L^{\infty}},\|\nabla u\|_{L^{\infty}}\right)$, such that:

$$
\begin{aligned}
& \|F(x, u)\|_{X^{k}} \leq C_{k}\left(1+\|u\|_{X^{k}}^{k}\right) \\
& \|G(x, u, v, \nabla u)\|_{X^{k-1}} \leq C_{k}^{\prime}\left(1+\|u\|_{X^{k}}^{k-1}+\|v\|_{X^{k-1}}\right) .
\end{aligned}
$$

The basis for the induction is incuded in the following

Proposition 2.1. There exists a constant $C>0$ independent of $\varepsilon \rightarrow 0$, such that:

$$
\begin{aligned}
& \|u\|_{X^{1}}+\|v\|_{X^{1}}+\|w\|_{X^{0}} \leq C \\
& \|u\|_{L^{\infty}} \leq C \\
& \|\nabla u\|_{L^{\infty}} \leq C
\end{aligned}
$$

In order to prove Proposition 2.1 we need some preliminary estimates.

Lemma 2.2. The following estimates hold, pointwise on $\Sigma$ :

$$
\begin{aligned}
& f(0) \leq f\left(e^{\widetilde{u}}\right) \leq s \\
& f(0) \leq v \leq s .
\end{aligned}
$$

Proof. By maximum principle. The increasing monotonicity of $f$ is essential here. 
As a consequence of Lemma 2.2, the nonlinearity $f$ may be truncated. Therefore in what follows, without loss of generality, we assume that:

$$
\sup _{t>0}\left\{|f(t)|+\left|f^{\prime}(t)\right|+\left|f^{\prime \prime}(t)\right|\right\} \leq C .
$$

The next identity is the main step in deriving the $H^{1}$-estimate for $v$ and the $L^{2}$-estimate for $w$ :

Lemma 2.3. The following identity holds:

$$
\int|\nabla v|^{2}+\int w^{2}=\int(s-v)\left(f^{\prime \prime}\left(e^{\widetilde{u}}\right) e^{\widetilde{u}}+f^{\prime}\left(e^{\widetilde{u}}\right)\right) e^{\widetilde{u}}|\nabla \widetilde{u}|^{2} .
$$

Proof. We compute:

$$
\Delta f\left(e^{\widetilde{u}}\right)=\left(f^{\prime \prime}\left(e^{\widetilde{u}}\right) e^{\widetilde{u}}+f^{\prime}\left(e^{\widetilde{u}}\right)\right) e^{\widetilde{u}}|\nabla \widetilde{u}|^{2}+f^{\prime}\left(e^{\widetilde{u}}\right) e^{\widetilde{u}} \Delta \widetilde{u} .
$$

Therefore $f\left(e^{\widetilde{u}}\right)$ satisfies the equation:

$$
-\Delta f\left(e^{u}\right)+\varepsilon^{-1} f^{\prime}\left(e^{\widetilde{u}}\right) e^{\widetilde{u}} f\left(e^{\widetilde{u}}\right)=\varepsilon^{-1} f^{\prime}\left(e^{\widetilde{u}}\right) e^{\widetilde{u}} v-\left(f^{\prime \prime}\left(e^{\widetilde{u}}\right) e^{\widetilde{u}}+f^{\prime}\left(e^{\widetilde{u}}\right)\right) e^{\widetilde{u}}|\nabla \widetilde{u}|^{2} .
$$

Integrating (17), we obtain

$$
\varepsilon^{-1} \int f^{\prime}\left(e^{\widetilde{u}}\right) e^{\widetilde{u}}\left(v-f\left(e^{\widetilde{u}}\right)\right)=\int\left(f^{\prime \prime}\left(e^{\widetilde{u}}\right) e^{\widetilde{u}}+f^{\prime}\left(e^{\widetilde{u}}\right)\right) e^{\widetilde{u}}|\nabla \widetilde{u}|^{2}
$$

Now we multiply (7) by $v-f\left(e^{\widetilde{u}}\right)$ and integrate to obtain:

$$
\int-\Delta v\left(v-f\left(e^{\widetilde{u}}\right)\right)=\varepsilon^{-1} \int f^{\prime}\left(e^{\widetilde{u}}\right) e^{\widetilde{u}}(s-v)\left(v-f\left(e^{\widetilde{u}}\right)\right)-\varepsilon^{-2} \int\left(v-f\left(e^{\widetilde{u}}\right)\right)^{2} .
$$

Integrating by parts and using (17) we find:

$$
\begin{aligned}
& \int-\Delta v\left(v-f\left(e^{\widetilde{u}}\right)\right)=\int|\nabla v|^{2}+\int v \Delta f\left(e^{\widetilde{u}}\right) \\
& =\int|\nabla v|^{2}-\varepsilon^{-1} \int v f^{\prime}\left(e^{\widetilde{u}}\right) e^{\widetilde{u}}\left(v-f\left(e^{\widetilde{u}}\right)\right)+\int v\left(f^{\prime \prime}\left(e^{\widetilde{u}}\right) e^{\widetilde{u}}+f^{\prime}\left(e^{\widetilde{u}}\right)\right) e^{\widetilde{u}}|\nabla \widetilde{u}|^{2} .
\end{aligned}
$$

Equating left hand sides in the last two identities, we obtain

$$
\begin{gathered}
\int|\nabla v|^{2}+\varepsilon^{-2} \int\left(v-f\left(e^{\widetilde{u}}\right)\right)^{2}+\int v\left(f^{\prime \prime}\left(e^{\widetilde{u}}\right) e^{\widetilde{u}}+f^{\prime}\left(e^{\widetilde{u}}\right)\right) e^{\widetilde{u}}|\nabla \widetilde{u}|^{2} \\
=s \varepsilon^{-1} \int f^{\prime}\left(e^{\widetilde{u}}\right) e^{\widetilde{u}}\left(v-f\left(e^{\widetilde{u}}\right)\right),
\end{gathered}
$$

and thus identity (16) is established.

We shall need some a priori estimates for solutions to

$$
-\varepsilon^{2} \Delta u+(1+\varepsilon c) u=f .
$$

Indeed, both (13) and (14) are of the form (19). 
Lemma 2.4. Let $c, f \in X^{k}$ and suppose that u satisfies: (19). For every $k \geq 0$ there exist $\varepsilon_{k}>0, C_{k}>0$ such that

$$
\|u\|_{X^{k}} \leq C_{k}\|f\|_{X^{k}},
$$

for all $\varepsilon \leq \varepsilon_{k}$.

Proof. The proof is an easy consequence of the following fact Let $G_{\varepsilon}$ be the Green's function for

$$
-\varepsilon \Delta_{x} G_{\varepsilon}+G_{\varepsilon}=\delta_{y} \quad \text { on } \Sigma .
$$

Then $G_{\varepsilon}(x, y) \rightarrow \delta_{y}$ weakly in the sense of measures. Note that since the operator $-\varepsilon \Delta+1$ is coercive, the Green's function $G_{\varepsilon}$ is uniquely defined on $\Sigma$. By the maximum principle, $G_{\varepsilon}>0$ on $\Sigma$. Integrating over $\Sigma$, we find $\int G_{\varepsilon}=\int\left|G_{\varepsilon}\right|=1$. Therefore, there exists a Radon measure $\mu$ such that $G_{\varepsilon} \neg \mu$ weakly in the sense of measures. For any $\varphi \in C^{\infty}(\Sigma)$ we have:

$$
\varepsilon \int-\Delta G_{\varepsilon} \varphi+\int G_{\varepsilon} \varphi=\varphi(y) .
$$

Taking limits, we find $\int \varphi \mathrm{d} \mu=\varphi(y)$ and the statement of the lemma is established.

Now we can provide the

Proof of Proposition 2.1. We begin by establishing

$$
\int e^{\widetilde{u}}|\nabla \widetilde{u}|^{2} \leq C .
$$

Proof of the Claim. Multiplying equation (6) by $e^{\widetilde{u}}$ and integrating by parts, we obtain

$$
\varepsilon^{-1} \int e^{\widetilde{u}}\left(v-f\left(e^{\widetilde{u}}\right)\right)=\int e^{\widetilde{u}}|\nabla \widetilde{u}|^{2} \geq 0 .
$$

By the pointwise estimates in Lemma 2.2, it follows that:

$$
\varepsilon \int e^{\widetilde{u}}|\nabla \widetilde{u}|^{2} \leq C
$$

Multiplying (7) by $e^{\widetilde{u}}$ and integrating, we find

$$
\varepsilon^{-1} \int e^{\widetilde{u}}\left(v-f\left(e^{\widetilde{u}}\right)\right)=\int e^{2 \widetilde{u}} f^{\prime}\left(e^{\widetilde{u}}\right)(s-v)+\varepsilon \int e^{\widetilde{u}} \Delta v .
$$

Integration by parts yields:

$$
\varepsilon \int e^{\widetilde{u}} \Delta v=-\int v e^{\widetilde{u}}\left(v-f\left(e^{\widetilde{u}}\right)\right)+\varepsilon \int v e^{\widetilde{u}}|\nabla \widetilde{u}|^{2} .
$$

Hence, by the pointwise estimates as in Lemma 2.2, and taking into account (21), we conclude that

$$
\varepsilon\left|\int e^{\widetilde{u}} \Delta v\right| \leq C
$$


Inserting into (22), recalling Lemma 2.2, we derive that

$$
\varepsilon^{-1} \int e^{\widetilde{u}}\left(v-f\left(e^{\widetilde{u}}\right)\right) \leq C
$$

and thus it follows that

$$
\int e^{\widetilde{u}}|\nabla \widetilde{u}|^{2}=\varepsilon^{-1} \int e^{\widetilde{u}}\left(v-f\left(e^{\widetilde{u}}\right)\right) \leq C .
$$

(20) is established.

Lemma 2.2 readily implies $\left\|e^{\widetilde{u}}\right\|_{L^{\infty}} \leq C$ and $\|v\|_{L^{\infty}} \leq C$. In order to obtain the $H^{1}$-estimate for $e^{\widetilde{u}}$, it suffices to observe that by Lemma 2.2-(i) and by (20) we have:

$$
\int\left|\nabla e^{\widetilde{u}}\right|^{2}=\int e^{2 \widetilde{u}}|\nabla \widetilde{u}|^{2} \leq C \int e^{\widetilde{u}}|\nabla \widetilde{u}|^{2} \leq C .
$$

Now we estimate $\|\nabla v\|_{L^{2}}$ and $\left\|\varepsilon^{-1}\left(v-f\left(e^{\widetilde{u}}\right)\right)\right\|_{L^{2}}$. Using identity (16), we have:

$$
\begin{aligned}
\int|\nabla v|^{2}+\int w^{2} & \leq\|s-v\|_{\infty}\left\|f^{\prime \prime}\left(e^{\widetilde{u}}\right) e^{\widetilde{u}}+f(\widetilde{u})\right\|_{\infty} \int e^{\widetilde{u}}|\nabla \widetilde{u}|^{2} \\
& \leq C \int e^{\widetilde{u}}|\nabla \widetilde{u}|^{2} \leq C,
\end{aligned}
$$

where we again used Lemma 2.2 and (20) in order to derive the last step. Proof of (i). Multiplying (9) by $u-\int u$ and integrating, we have:

$$
\begin{aligned}
\int|\nabla u|^{2} & =q \int\left(v-f\left(e^{\widetilde{u}}\right)\right)\left(u-\int u\right) \\
& \leq\left\|q\left(v-f\left(e^{\widetilde{u}}\right)\right)\right\|_{2}\left\|u-\int u\right\|_{2} \leq C\|\nabla u\|_{2},
\end{aligned}
$$

where the last inequality follows by Lemma 2.2 and by the Poincaré inequality. Hence $\|\nabla u\|_{2} \leq C$. By Lemma $2.2-$ (ii), we have that $e^{\widetilde{u}} \leq C$, and thus we only have to show that $\int u \geq-C$. To this end, we first observe that integrating (9) and (10) we obtain:

$$
\int f^{\prime}\left(e^{u_{0}+u}\right) e^{u_{0}+u}(s-v)=q \int\left(v-f\left(e^{u_{0}+u}\right)\right)=4 \pi n .
$$

On the other hand, we have in a straightforward manner:

$$
\int f^{\prime}\left(e^{u_{0}+u}\right) e^{u_{0}+u}(s-v) \leq C \int e^{u_{0}+u} \leq C e^{\int u}\left\|e^{u_{0}}\right\|_{\infty} \int e^{u-\int u} \leq C \int e^{u-\int u} .
$$

Hence, recalling the Moser-Trudinger inequality (see [1]) and the estimate for $\|\nabla u\|_{2}$, we conclude that

$$
4 \pi n \leq C e^{\int u} \int e^{u-\int u} \leq C e^{\int u} e^{\gamma \int|\nabla u|^{2}} \leq C e^{\int u},
$$

which establishes (i). Proof of (ii). Since $\|w\|_{L^{2}} \leq C$, by (i) and elliptic regularity we obtain $\|u\|_{H^{2}} \leq C$. Then Sobolev embeddings yield $\|\nabla u\|_{L^{p}} \leq C$, for any $1 \leq p<+\infty$ and $\|u\|_{L^{\infty}} \leq C$, which establishes (ii). Proof of (iii). By (14), $\|\nabla u\|_{L^{p}} \leq C$ and Lemma 2.4 imply that $\|w\|_{L^{p}} \leq C$, for any $1 \leq p<+\infty$. Then (12) and Sobolev embeddings yield $\|u\|_{W^{2, p}} \leq C$, for any $1 \leq p<+\infty$. For $p>2$, the Sobolev embeddings yield (iii). 
Proposition 2.2. For all $k \geq 0$ there exists a constant $C>0$ (possibly depending on $k$ ) such that:

$$
\left\|\widetilde{u}-u_{0}\right\|_{H^{k}}+\|v\|_{H^{k}} \leq C .
$$

Proof of Proposition 2.2. We argue by induction on $k \in \mathbb{N}_{0}$.

CLAIM: Suppose:

$$
\|u\|_{X^{k}}+\|v\|_{X^{k}}+\|w\|_{X^{k-1}} \leq C_{k} .
$$

Then:

$$
\|u\|_{X^{k+1}}+\|v\|_{X^{k+1}}+\|w\|_{X^{k}} \leq C_{k+1} .
$$

Indeed,

$$
\begin{aligned}
\|w\|_{X^{k-1}} \leq C & \Rightarrow\|u\|_{X^{k+1}} \leq C & & \text { by }(12) \text { and standard elliptic regularity } \\
& \Rightarrow\|v\|_{X^{k+1}} \leq C & & \text { by (13), Lemma } 2.1 \text { and Lemma } 2.4 \\
& \Rightarrow\|w\|_{X^{k}} \leq C & & \text { by (14), Lemma } 2.1 \text { and Lemma } 2.4 .
\end{aligned}
$$

Now Proposition 2.1, the Claim and a standard induction argument conclude the proof.

Finally, we can prove our main result:

Proof of Theorem 1.3. Let $(u, v)$ be solutions to system (9)-(10), with $\varepsilon \rightarrow$ 0 . By the a priori estimates as stated in Proposition 2.2 and by standard compactness arguments, there exist $u_{\infty}, v_{\infty}$ such that up to subsequences $u \rightarrow$ $u_{\infty}$ and $v \rightarrow v_{\infty}$ in $C^{h}$, for all $h \geq 0$. We write (9) in the form:

$$
v=f\left(e^{u_{0}+u}\right)+\varepsilon(-\Delta u+4 \pi n) .
$$

Taking limits, we find $v_{\infty}=f\left(e^{u_{0}+u_{\infty}}\right)$. Furthermore, taking limits in (10), we obtain

$$
\varepsilon^{-1}\left(v-f\left(e^{u_{0}+u}\right)\right) \rightarrow f^{\prime}\left(e^{u_{0}+u_{\infty}}\right) e^{u_{0}+u_{\infty}}\left(s-f\left(e^{u_{0}+u_{\infty}}\right)\right),
$$

where the convergence holds in $C^{h}$, for any $h \geq 0$. Consequently, taking limits in (9), we find that $u_{\infty}$ satisfies:

$$
-\Delta u_{\infty}=f^{\prime}\left(e^{u_{0}+u_{\infty}}\right) e^{u_{0}+u_{\infty}}\left(s-f\left(e^{u_{0}+u_{\infty}}\right)\right)-4 \pi \sum_{j=1}^{n} \delta_{p_{j}} .
$$

Setting $\widetilde{u}_{\infty}=u_{0}+u_{\infty}$, we conclude the proof of Theorem 1.3.

\section{Acknowledgement}

I should like to thank the Organizers of the ICM2002 Satellite Conference on Nonlinear Functional Analysis, Taiyuan, August 14-18, as well as Professor Yanyan Li, for the invitation. 


\section{References}

[1] Th. Aubin, Nonlinear Analysis on Manifolds: Monge-Ampère Equations, Springer-Verlag, New York, Berlin, 1982

[2] D. Chae and N. Kim, Vortex Condensates in the Relativistic Self-Dual Maxwell-Chern-Simons-Higgs System, preprint

[3] D. Chae and H.S. Nam, On the Condensate Multivortex Solutions of the Self-Dual Maxwell-Chern-Simons $C P(1)$ Model, Ann. H. Poincaré 2 (2001), 887-906

[4] K. Kimm, K. Lee and T. Lee, Anyonic Bogomol'nyi Solitons in a Gauged $O$ (3) Sigma Model, Phys. Rev. D 53 (1996), 4436-4440

[5] G. Dunne, Self-Dual Chern-Simons Theories, Lecture Notes in Physics, vol. M36, Springer-Verlag, Berlin, New York, 1995

[6] J. Hong, Y. Kim and P.Y. Pac, Multivortex solutions of the Abelian Chern-Simons theory, Phys. Rev. Lett. 64 (1990), 2230-2233

[7] R. Jackiw and E. Weinberg, Self-dual Chern-Simons vortices, Phys. Rev. Lett. 64 (1990), 2234-2237

[8] A. Jaffe, C. Taubes, Vortices and Monopoles, Birkhäuser, Boston, 1980

[9] C. Lee, K. Lee and H. Min, Self-Dual Maxwell Chern-Simons Solitons, Phys. Lett. B, 252 (1990), 79-83

[10] L. Nirenberg, On elliptic partial differential equations, Ann. Sc. Norm. Sup. Pisa, 13 (1959), 116-162

[11] T. Ricciardi, Asymptotics for Maxwell-Chern-Simons multivortices, Nonlin. Anal. T.M.A. 50 No. 8 (2002), 1093-1106.

[12] T. Ricciardi, On a nonlinear elliptic system from Maxwell-Chern-Simons vortex theory, preprint.

[13] T. Ricciardi and G. Tarantello, Vortices in the Maxwell-Chern-Simons Theory, Comm. Pure Appl. Math. 53 no. 7 (2000), 811-851

[14] Y. Yang, Solitons in field theory and nonlinear analysis, Springer Monographs in Mathematics, Springer-Verlag, New York, 2001

http://cds.unina.it/tonricci 